\title{
Drag Behavior of an Inflatable Reentry Vehicle in the Transonic Regime
}

\author{
Yusuke Takahashi ${ }^{1}$, Manabu Matsunaga $^{2}$, Nobuyuki Oshima $^{3}$ \\ Hokkaido University, Kita 13 Nishi 8, Kita-ku, Sapporo, Hokkaido 060-8628, Japan \\ and \\ Kazuhiko Yamada ${ }^{4}$ \\ Japan Aerospace Exploration Agency, 3-1-1 Yoshinodai Chuo-ku, Sagamihara, Kanagawa \\ 252-5210, Japan
}

\begin{abstract}
The drag coefficient for inflatable reentry vehicles shows discrepancies between a wind tunnel experiment and a flight test in a transonic regime. These discrepancies exist in the drag decrease behavior in the transonic regime and local minimum values of drag above the sonic speed in a wind tunnel. Hence, the present paper focuses on uncovering the reasons and mechanisms behind the same and investigates transonic flow fields around a vehicle by using a transonic wind tunnel and the computational fluid dynamics (CFD) approach. Several test models with diameters ranging from 56 to $96 \mathrm{~mm}$ are used to quantitatively evaluate the effects of scale and a sting attached on the rear. Aerodynamic coefficients, pressures at the rear of the model, and density gradient distributions are measured for operation conditions of free-stream Mach numbers ranging between 0.8 and 1.3. In addition, detailed distributions of the flow field properties are clarified using the CFD method, which is validated by the experimental data. The results indicate that a sting behind the test models reduces the steep drag decrease at transonic speeds and that shock waves reflected on the test-section walls of the wind tunnel result in local minimum values at supersonic speeds.
\end{abstract}

\section{Nomenclature}

$C=$ coefficient

$D=\operatorname{drag}, \mathrm{N}$

$\mathrm{M}=$ Mach number

$\mathrm{nm}=$ number of molecules

$\mathrm{ns}=$ number of species

$p \quad=$ pressure, $\mathrm{Pa}$

$q=$ dynamic pressure, $\mathrm{Pa}$

$R=$ specific gas constant, $\mathrm{J} /(\mathrm{kg} \cdot \mathrm{K})$

Re $=$ Reynolds number

$S=$ area, $\mathrm{m}^{2}$

$T=$ temperature, $\mathrm{K}$

$u=$ velocity, $\mathrm{m} / \mathrm{s}$

$x=$ coordinate, $\mathrm{m}$

$\delta_{i j}=$ Kronecker's delta

$\lambda=$ thermal conductivity, $\mathrm{W} /(\mathrm{m} \cdot \mathrm{K})$

\footnotetext{
${ }^{1}$ Assistant Professor, Faculty of Engineering; ytakahashi@eng.hokudai.ac.jp.

${ }^{2}$ Graduate student, Graduate School of Engineering, (Currently: IHI Corporation)

${ }^{3}$ Professor, Faculty of Engineering.

${ }^{4}$ Associate Professor, Institute of Space and Astronautical Science.
} 


$$
\begin{aligned}
\mu & =\text { viscosity, } \mathrm{Pa} \cdot \mathrm{s} \\
\rho & =\text { density, } \mathrm{kg} / \mathrm{m}^{3} \\
\tau_{i j} & =\text { stress tensor, } \mathrm{Pa}
\end{aligned}
$$

$$
\begin{aligned}
& \text { Subscripts } \\
& \begin{array}{l}
D=\text { drag } \\
p=\text { pressure } \\
s \quad=\text { chemical species } \\
\mathrm{t}=\text { turbulence } \\
\infty \quad=\text { free stream }
\end{array}
\end{aligned}
$$

\section{Introduction}

The flare-type thin-membrane inflatable reentry vehicle technology has been developed, and has been found to have several advantages. Specifically, it provides a reduction in aerodynamic heating during atmospheric reentry [1-7]. Extant elemental studies and demonstration flights have been conducted in the framework of the Membrane Aeroshell for Atmosphericentry Capsule (MAAC) project [8-12].

A reentry demonstration was conducted on August 7, 2012, by using an S-310-41 sounding rocket (SMAAC: Sounding Rocket Experiment of Membrane Aeroshell for Atmospheric-Entry Capsule) at the Uchinoura Space Center, Kagoshima, Japan [13-18]. The main purpose of this study involved demonstrating a low-ballistic-coefficient flight of a type of inflatable vehicle during suborbital reentry. The flight vehicle mainly consists of three components: a capsule, a membrane aeroshell, and an inflatable torus. Figures 1 and 2 show schematics of SMAAC and the reentry trajectory of the flight demonstration test, respectively. The overall diameter of SMAAC was 1,200 mm and the total mass was $15.6 \mathrm{~kg}$. The peak velocity was approximately $1,320 \mathrm{~m} / \mathrm{s}$ at an altitude of $66 \mathrm{~km}$. SMAAC continued to fly with a terminal velocity of $15 \mathrm{~m} / \mathrm{s}$ below an altitude of $23 \mathrm{~km}$, and finally, splashed down in the Pacific Ocean. Though SMAAC itself was not recovered, all flight data measured throughout the mission were obtained by telemetry.

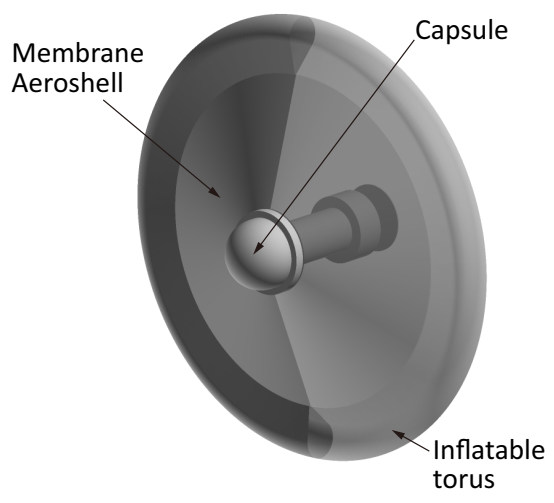

Figure 1: Schematic of SMAAC.

The drag coefficient profile in a free flight environment was obtained in the suborbital reentry mission. Steep decreases in the drag coefficient were confirmed with decreases in the 


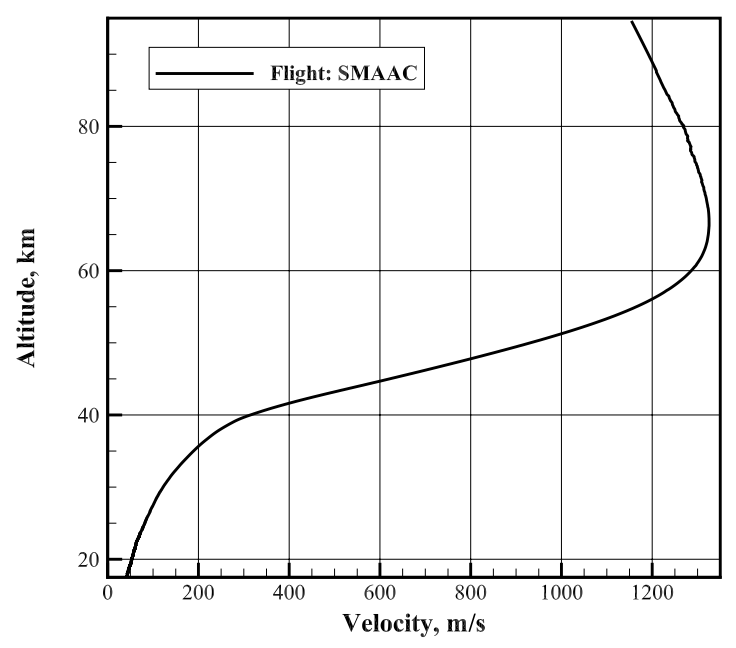

Figure 2: Reentry trajectory of SMAAC flight test.

Mach number in the transonic regime. The impact of rear pressure on aerodynamic characteristics is emphasized in the transonic regime because the rear of an inflatable reentry vehicle generally corresponds to a large area. The wake structure behind the vehicle is expected to be important. As a preliminary study before the SMAAC demonstration test, drag profiles relative to the Mach number in the transonic regime were investigated using the transonic wind tunnel of the Institute of Space and Astronautical Science at the Japan Aerospace Exploration Agency (ISAS/JAXA). Discrepancies were found in the drag coefficient profiles between the wind tunnel test and the SMAAC flight test in the transonic regime. This indicates that the wind tunnel test for such inflatable vehicles insufficiently reproduces the flight environment. Although the transonic wind tunnel is an effective ground-based facility to investigate aerodynamics and attitude for reentry vehicles, its insufficient reproduction of the flight environment can be a fatal problem for a mission that uses an inflatable reentry vehicle. It is necessary to clarify the reasons and mechanisms of the discrepancies for more reliable tests by using a wind tunnel and to evaluate accurate aerodynamic characteristics.

Besides the wind tunnel experiment, the computational fluid dynamics (CFD) approach is also effective in investigating the flow field in detail. Aerodynamics and aerodynamic heating for SMAAC were investigated using measurement data and CFD $[18,19]$. The results suggested that the CFD technique adequately predicts the complicated flow field around SMAAC. It is important to clarify the drag coefficient behavior at transonic speeds observed in the flight test. Hence, in this study, the research objectives included reproducing the environment of the wind tunnel test with a CFD technique and investigating the discrepancies between the wind tunnel experiment and flight data.

\section{Wind Tunnel}

\section{$2.1 \quad$ Facility}

The experiments are performed by using the JAXA/ISAS transonic wind tunnel facility. The facility corresponds to a blow-down type wind tunnel. The capacity and maximum gauge 
pressure in the reservoir correspond to $1,767 \mathrm{~m}^{3}$ and $931.6 \mathrm{kPa}$, respectively. The time duration of flow exceeds $30 \mathrm{~s}$. The Mach number range is from 0.3 to 1.3. Ranges of the angle of attack and angle of sideslip correspond to \pm 17 degrees. The facility has a square test section with a side length of $0.6 \mathrm{~m}$. As the wall of the test section is porous to reduce reflection of wave and blockage effect, the aforementioned problem does not normally appear in an aerodynamic study. A test model is normally supported by a sting in the test section.

\section{$2.2 \quad$ Test Models}

To investigate the scale effect during the experiment, five test models with different diameters corresponding to $96,86,76,66$, and $56 \mathrm{~mm}$ are composed of aluminum, as shown in Fig. 3. Although the size of the test model set is variable, the shape of the sting is fixed. Figure 4 shows a cross-section shape of the test model with a diameter of $96 \mathrm{~mm}$. All dimensions are given in millimeters. Curves of the aeroshell part are as described in Fig. 5, and are given by the results computed based on a particle method [20]. The aeroshell shapes of the test models are close to that of the flight test. Shapes of the aeroshell are similar among these test models. However, tops of all test models are fixed with a semi-sphere shape with $16 \mathrm{~mm}$ diameter and the shapes of the torus tube are also fixed; therefore, the similarity is imperfect with the exception of the models with $96 \mathrm{~mm}$ diameter. A sting with $45 \mathrm{~mm}$ diameter is attached on the rear of the test models.

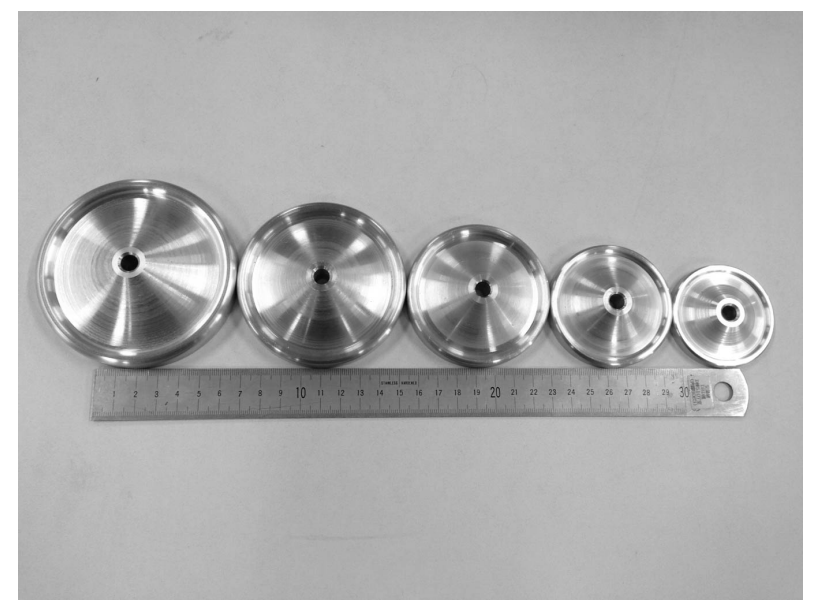

Figure 3: Test models with diameters of $96,86,76,66$, and $56 \mathrm{~mm}$ from the left side.

The rear side of the aforementioned test models corresponds to a cutout shape. However, the rear shape of SMAAC is not actually a cutout shape because of a thin membrane. To investigate the shape effect at the rear side, a "gouged-shaped" test model is also created as shown in Fig. 6. All shapes of the test model, except for the rear, are the same as the cutout-shaped test model with a diameter of $86 \mathrm{~mm}$.

\subsection{Measurements}

Aerodynamic forces on the test models are measured by using a six-element internal balance of the wind tunnel. To obtain pressure data in the wake of the test models, four static pressure tubes are set behind the test model as shown in Fig. 7. The four pressure tubes 


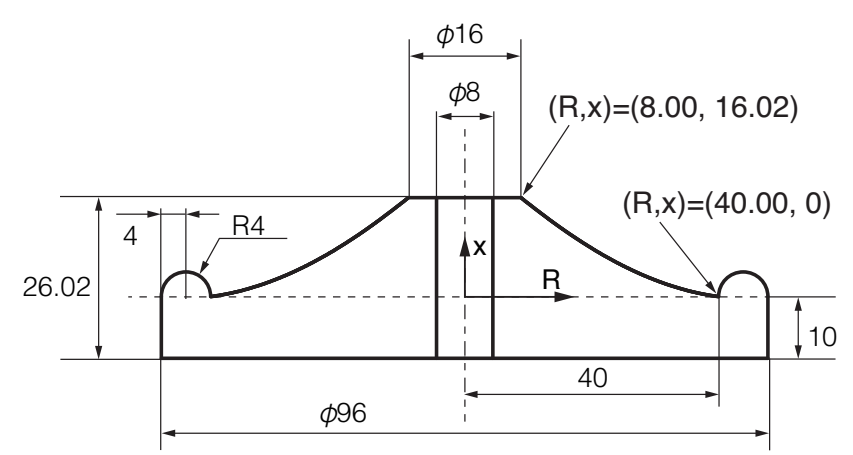

Figure 4: Shape of the test model with $96 \mathrm{~mm}$ diameter (dimension is in millimeters).

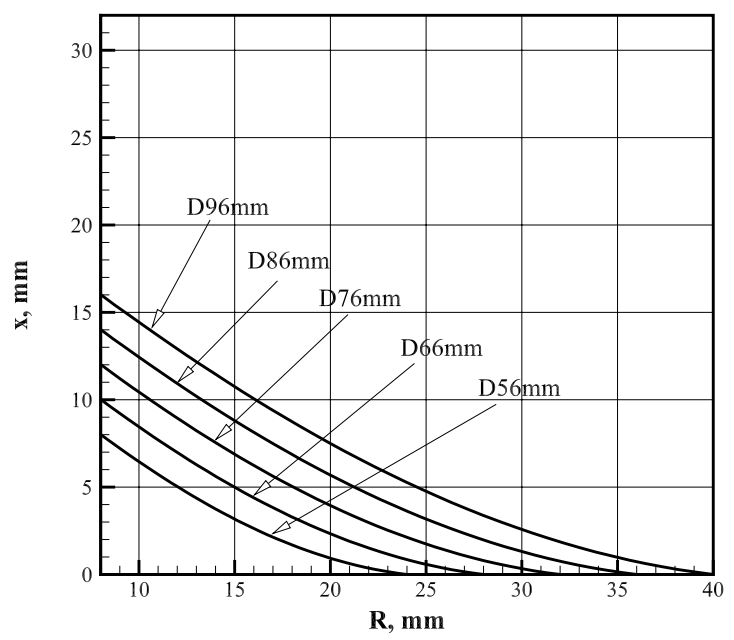

Figure 5: Coordinate of the aeroshell part of the test models.

(P1, P2, P3, and P4) are arranged at 90 degrees of phase in the circumferential direction. All distances between the rear of the test model and top of the pressure tubes correspond to $5 \mathrm{~mm}$. Additionally, color schlieren photography is used for visualization of the flow field around the test models. The schlieren method is used to measure density gradient of the flow field in a certain direction, and information is obtained on compression, expansion, and shock wave formation.

\subsection{Test Conditions}

Wind tunnel tests for the five test models are performed in the transonic regime. The freestream Mach number changes continuously from 1.3 to 0.8 over a time of $52 \mathrm{~s}$. The angle of attack is set to $0^{\circ}$ during the continuous variation of the Mach number. Additionally, the angle of attack is varied from -5 and 15 for a fixed Mach number. Aerodynamic forces, pressure in the wake, and density-gradient distribution are measured, and sampling frequencies of the balance and pressure tubes are set to $1,000 \mathrm{~Hz}$. 


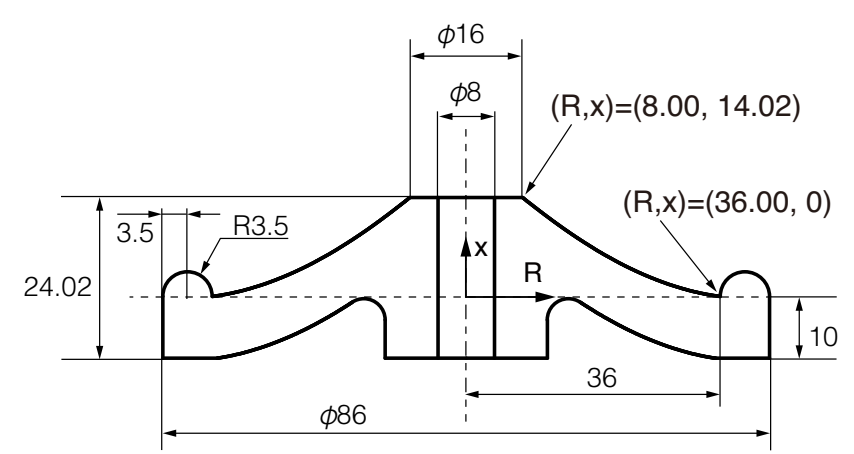

Figure 6: Shape of the gouged-type test model with $86 \mathrm{~mm}$ diameter (dimension is in millimeters).

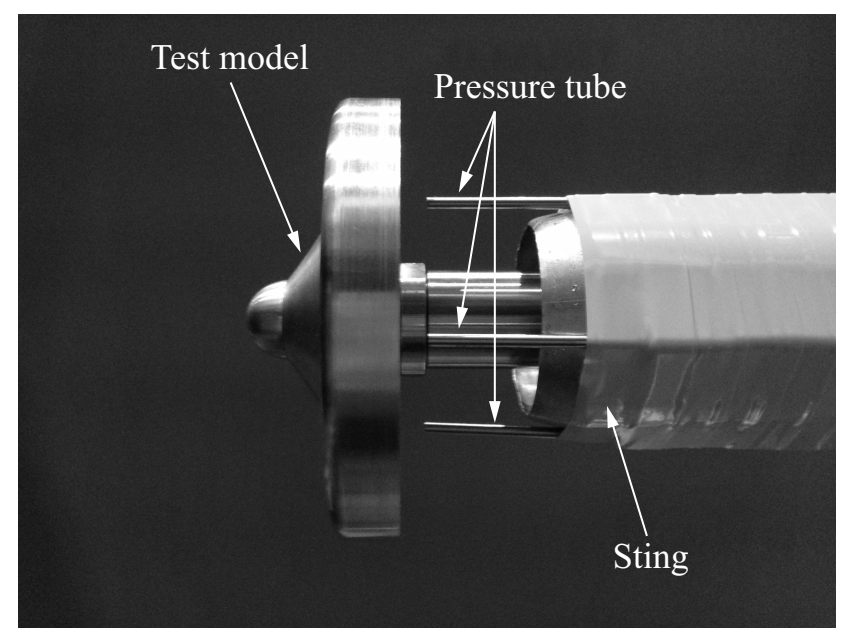

Figure 7: Test model, pressure tubes, and sting.

\section{Analytical Method}

The governing equations, physical models, and numerical implementation used in the present study are described in this section.

\subsection{Governing Equation}

The following assumptions are used in the analytical model: (1) The flow is continuum and turbulent; (2) The gas considered corresponds to air, which is composed of 0.765 of $\mathrm{N}_{2}$ and 0.235 of $\mathrm{O}_{2}$ in mass fraction; and (3) No chemical reaction occurs.

The Navier-Stokes (NS) equations and the equation of state describe the flow fields. The NS equations are composed of conservation equations for the total mass, momentum, and 
total energy and are expressed as follows:

$$
\begin{aligned}
& \frac{\partial \rho}{\partial t}+\frac{\partial}{\partial x_{j}}\left(\rho u_{j}\right)=0, \\
& \frac{\partial\left(\rho u_{j}\right)}{\partial t}+\frac{\partial}{\partial x_{j}}\left(\rho u_{i} u_{j}+\delta_{i j} p\right)=\frac{\partial \tau_{i j}}{\partial x_{j}}, \\
& \frac{\partial E}{\partial t}+\frac{\partial}{\partial x_{j}}\left[(E+p) u_{j}\right]=\frac{\partial}{\partial x_{j}}\left(u_{j} \tau_{i j}\right)+\frac{\partial q_{j}}{\partial x_{j}},
\end{aligned}
$$

where $\delta_{i j}$ denotes the Kronecker delta. Additionally, $\tau_{i j}$ and $q_{j}$ denote the stress tensor and heat flux, respectively, which are given as follows:

$$
\begin{aligned}
\tau_{i j} & =\mu\left(\frac{\partial u_{i}}{\partial x_{j}}+\frac{\partial u_{j}}{\partial x_{i}}-\frac{2}{3} \frac{\partial u_{k}}{\partial x_{k}} \delta_{i j}\right), \\
q_{j} & =\lambda \frac{\partial T}{\partial x_{j}} .
\end{aligned}
$$

The equation of state is expressed as follows:

$$
p=\sum_{s=1}^{\mathrm{ns}} \rho_{s} R_{s} T .
$$

The total energy includes translational, rotational, and vibration internal energies. The molecular viscosity is evaluated by using Sutherland's law. The thermal conductivity is calculated by using the viscosity and the Prandtl number that is set to 0.71 .

In order to express turbulent flow, the governing equations of the flow field are converted to Reynolds-averaged Navier-Stokes (RANS) equations by using the Favre-averaging manner. A turbulence model is introduced to express the Reynolds stress derived in this manner. The shear stress transport turbulence model 2003 version (SST2003) [21] is adopted for a turbulence model. The eddy viscosity is used to replace transport properties in the flow field equations in the following manner: (1) $\mu \rightarrow \mu+\mu_{\mathrm{t}}$, (2) $\lambda \rightarrow \lambda+\lambda_{\mathrm{t}}$. The turbulent heat conductivity, $\lambda_{\mathrm{t}}$, is evaluated by using the the turbulent viscosity, turbulent Prandtl number that is set to 0.90 , and specific heat at constant pressure.

\subsection{Numerical Implementation}

In the present study, RG-FaSTAR v2.1.0 [22] is adopted, and this corresponds to a highenthalpy flow solver that incorporates real gas effects and thermochemical nonequilibrium and is a version of the fast unstructured CFD code FaSTAR [23] that was originally developed by JAXA. The governing equations of the flow field are solved by using a finite-volume approach. All the flow properties are set at the center of a control volume. The advection fluxes in the NS equations are calculated by using the SLAU scheme [24] with the MUSCL interpolation method for high accuracy. The viscous fluxes are evaluated by using the second-order central difference method. The spatial gradients of the flow properties are calculated by using the Green-Gauss method. Time integration is performed by using an implicit time-marching method. The system of governing equations is transformed into the delta form, and the solution is updated at each time step. With respect to the time integration, the lower-upper symmetric Gauss-Seidel (LU-SGS) method is employed. With respect to the massive parallel computation on a high-performance computer, the message passing interface (MPI) technique with a domain partition approach is used. 


\subsection{Computational Grids}

Figure 8 shows the computational domain of the test model with a diameter of $86 \mathrm{~mm}$. Essentially a similar computational domain is also used with respect to the analytical cases without a sting model. The computational domain includes the inlet boundary in front of the test model, outlet boundary in the rear of the model, and wall boundary on the surface of the model. At the inlet boundary, the free-stream parameters are given as subsequently mentioned. The nonslip condition for the velocity is imposed at the surfaces. Additionally, no pressure gradient normal to the surface is assumed. The temperature is fixed to $300 \mathrm{~K}$ at the surface. At the outlet boundary, the gradient-free condition for the flow parameters is imposed.

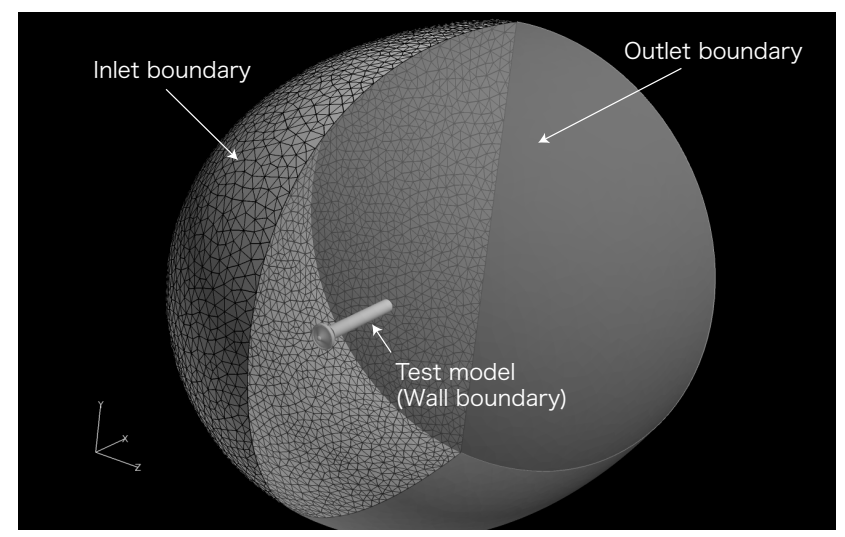

Figure 8: Computational domain for the test model with $86 \mathrm{~mm}$ diameter.

The wall effect of the wind tunnel is expected to be required for a more accurate prediction for the transonic wind tunnel. However, given the scope of the present research objectives, it is sufficient to only reproduce the qualitative trend of the wall effect.

The computational grid systems shown in Figs. 9(a) and 9(b) are used in the simulation cases of the test model with $86 \mathrm{~mm}$ diameter with a sting and without a sting, respectively. The numbers of cells and nodes for test models featuring a sting correspond to 5,407,415 and 963,363, respectively. Conversely, the numbers of cells and nodes for test models without a sting correspond to $3,264,672$ and 570,181, respectively. In the grid systems, only a tetrahedral mesh is used throughout the entire computational domain.

\subsection{Computational Conditions}

Inlet parameters, such as free stream density, temperature, and velocity, are calculated by using isentropic relations based on the reservoir conditions (pressure and temperature) and with a given Mach number and specific heat ratio. It is assumed that the reservoir pressure, reservoir temperature, and specific heat ratio correspond to $150 \mathrm{kPa}, 300 \mathrm{~K}$, and 1.402 , respectively, and free stream conditions imposed on the inlet boundary of the computational domain are obtained as listed in Table 1 . The Reynolds number is calculated by using a diameter of $86 \mathrm{~mm}$ of the test model as the characteristic length and viscosity as obtained by the Sutherland's law. 


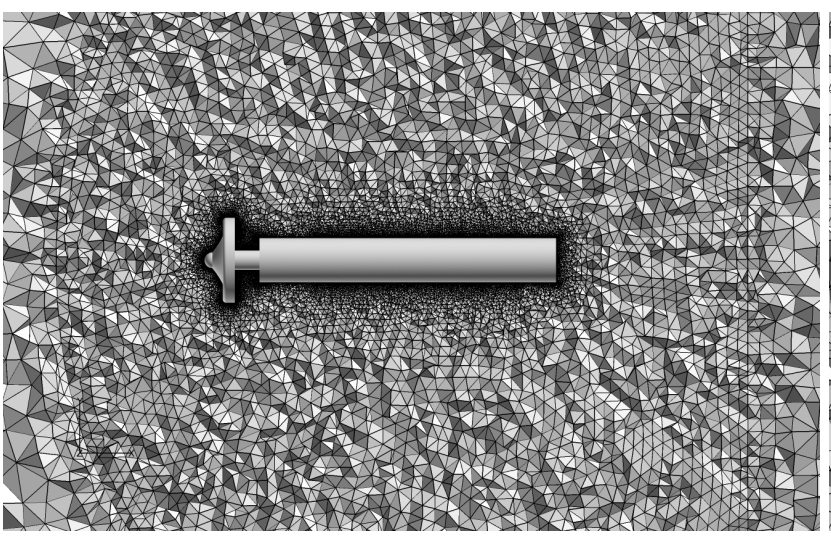

(a) Case with sting

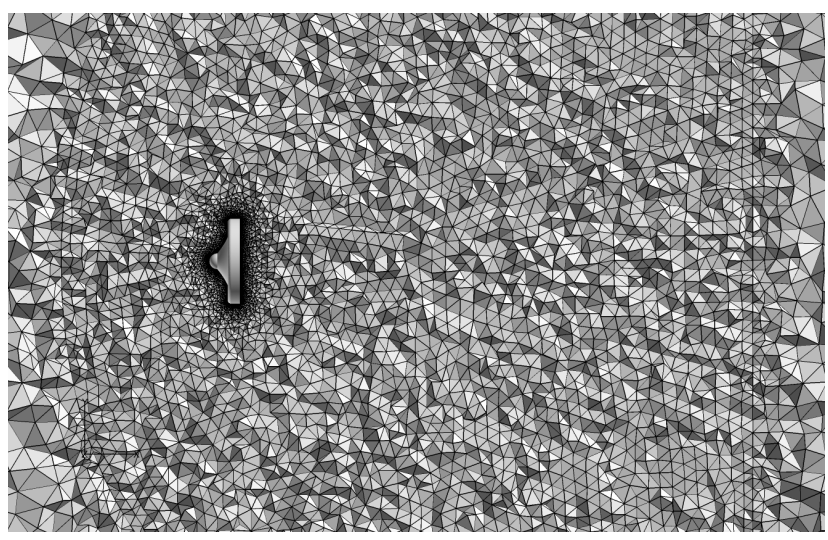

(b) Case without sting.

Figure 9: Computational grid systems around the test model with $86 \mathrm{~mm}$ diameter.

Table 1: Free-stream conditions at the inlet boundary.

\begin{tabular}{ccccc}
\hline \hline $\begin{array}{c}\text { Mach number, } \\
-\end{array}$ & $\begin{array}{c}\text { Density, } \\
\mathrm{kg} / \mathrm{m}^{3}\end{array}$ & $\begin{array}{c}\text { Temperature, } \\
\mathrm{K}\end{array}$ & $\begin{array}{c}\text { Velocity, } \\
\mathrm{m} / \mathrm{s}\end{array}$ & $\begin{array}{c}\text { Reynolds number, } \\
(\mathrm{D} 86 \mathrm{~mm})\end{array}$ \\
\hline 0.8 & 1.289 & 265.8 & 261.7 & $1.73 \times 10^{6}$ \\
0.9 & 1.197 & 258.0 & 290.0 & $1.83 \times 10^{6}$ \\
1.0 & 1.104 & 249.8 & 317.1 & $1.90 \times 10^{6}$ \\
1.1 & 1.013 & 241.3 & 342.8 & $1.94 \times 10^{6}$ \\
1.2 & 0.925 & 232.7 & 367.2 & $1.95 \times 10^{6}$ \\
1.3 & 0.841 & 223.9 & 390.3 & $1.95 \times 10^{6}$ \\
\hline \hline
\end{tabular}

\section{Results and Discussion}

\subsection{Aerodynamics}

The drag and rear pressures of the test model are obtained in a Mach number ranging between 0.8 and 1.3 by using the transonic wind tunnel. The drag coefficient, $C_{\mathrm{D}}$, and pressure coefficient, $C_{\mathrm{p}}$, are defined as follows:

$$
\begin{aligned}
C_{\mathrm{D}} & =\frac{D}{q_{\infty} S}, \\
C_{\mathrm{p}} & =\frac{p-p_{\infty}}{q_{\infty}},
\end{aligned}
$$

where $q_{\infty}$ and $p_{\infty}$ denote the dynamic and static pressures of the free stream, respectively. In addition, $S$ is the characteristic area (that corresponds to each front-projected area of the test models).

The relative measurement uncertainties of the drag coefficient and the pressure coefficients of the four rear probes are shown in Table 2. These are the results of the case for the test model with $96 \mathrm{~mm}$ diameter for no angle of attack at a fixed Mach number of 1 . The uncertainty is evaluated as the standard deviation here.

Figures 10 and 11 show the drag and pressure coefficient profiles, respectively, relative to the Mach number of the free stream for no angle of attack. In Fig. 10, the drag coefficient 
Table 2: Relative measurement uncertainty.

\begin{tabular}{ccccc}
\hline \hline$C_{\mathrm{D}}$ & $C_{\mathrm{p}}(\mathrm{P} 1)$ & $C_{\mathrm{p}}(\mathrm{P} 2)$ & $C_{\mathrm{p}}(\mathrm{P} 3)$ & $C_{\mathrm{p}}(\mathrm{P} 4)$ \\
\hline $3.74 \%$ & $1.33 \%$ & $1.23 \%$ & $1.12 \%$ & $0.911 \%$ \\
\hline \hline
\end{tabular}

profile measured in the flight demonstration test (SMAAC) by using a sounding rocket [14] is also included as a solid line with white symbols for the purposes of comparison with the wind tunnel experiments. Note that the Reynold number in the SMAAC flight is approximately $10^{5}$ for the Mach number ranging 1.3-0.8. [25]. The wind tunnel data constitute the results of the cutout-shaped test models. The drag data are averaged in a moving-average manner during $0.1 \mathrm{~s}$. Conversely, the pressure data are arithmetically averaged using values obtained by the four pressure tubes in the rear of the test model. With respect to the three cases of the test models with diameters of 96,86 , and $76 \mathrm{~mm}$, operations are performed twice in Mach number ranges of $M=1.2-0.8$ and $M=1.3-1.1$. The drag and pressure profiles almost overlap in this range in the figures due to good reproducibility.

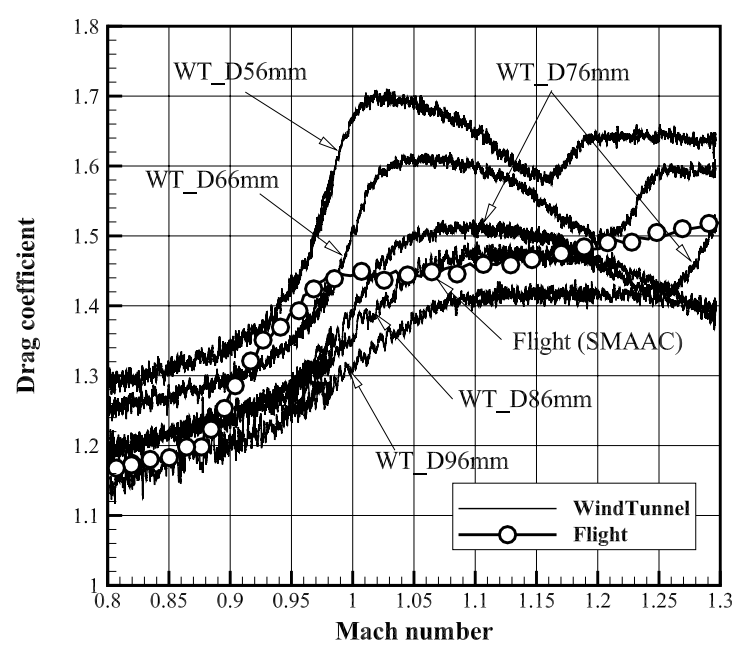

Figure 10: Drag coefficient profiles relative to the Mach number for different scale test model cases in the wind tunnel experiment and flight demonstration case.

The drag coefficient profile of the flight test gradually decreases when the Mach number decreases, while the profile exhibits a steep decrease below a Mach number that approximately corresponds to 1 . The drag coefficient profiles of the five test models in the wind tunnel experiments do not reproduce the steep decrease in the flight at the Mach number of 1 . When compared to the flight test, drag coefficients of the smaller model are overestimated, while those of the larger model are underestimated. The results confirm that the local minimum values of the drag coefficient for each test model appear in the supersonic region. Figure 11 shows increases in the rear pressure in the local minimum value region of the drag. The minimum and maximum values of the drag and rear pressures appear at the lower Mach number when the scale of the test model decreases. In the subsonic region, the rear pressure decreases and the drag coefficient profiles increase when the scale decreases. Thus, the drag 


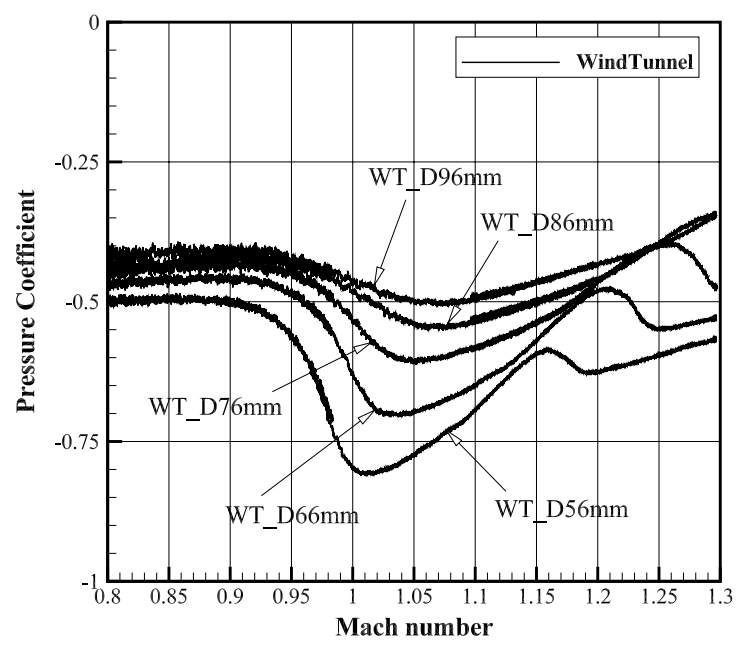

Figure 11: Pressure coefficient profiles relative to the Mach number for different scale test model cases in the wind tunnel experiment.

coefficients of the test models are strongly affected by the rear pressure.

The drag coefficient and rear pressure profiles of the gouged-shaped test model with 86 $\mathrm{mm}$ diameter are almost the same as the results of the cutout-shaped test model with 86 $\mathrm{mm}$ diameter, although graphs of the measurement data are not illustrated here. Thus, it is concluded that the shape effect at the rear of the test model is low in the transonic wind tunnel.

Drag and lift coefficients relative to the angle of attack for the test model with $86 \mathrm{~mm}$ diameter at the free-stream Mach number of 1.2 are shown in Fig. 12. It is indicated that the tendency of the drag against the angle of attack is small in this range, while the lift linearly changes.

\subsection{Shock Waves}

The color schlieren images around the test model with $66 \mathrm{~mm}$ diameter for Mach number conditions between 1.3 and 1.05 are shown in Fig. 13. The images indicate the distributions of the density gradient in the main stream direction ( $x$ direction), which is expressed as follows:

$$
\operatorname{Grad} . x(\rho)=\frac{\partial \rho}{\partial x}
$$

In the color schlieren technique, the region in which the density gradient is positive is denoted in red, while that in which the density gradient is negative is denoted in blue.

A strong shock wave in front of the test model and a large expansion region in its rear are observed in all cases. When the Mach number decreases, the shock stand-off distance increases and the front shock wave tends toward a normal shock. Oblique shock waves are generated on the sting surface. Note that a shock wave reflected on the wall of the wind tunnel facility attaches on the sting backward in the case where the Mach number corresponds to 1.30. The porous walls suppressing the shock wave reflection are ineffective for the present tests. The reflected shock waves continue to move forward to the sting at Mach numbers of 


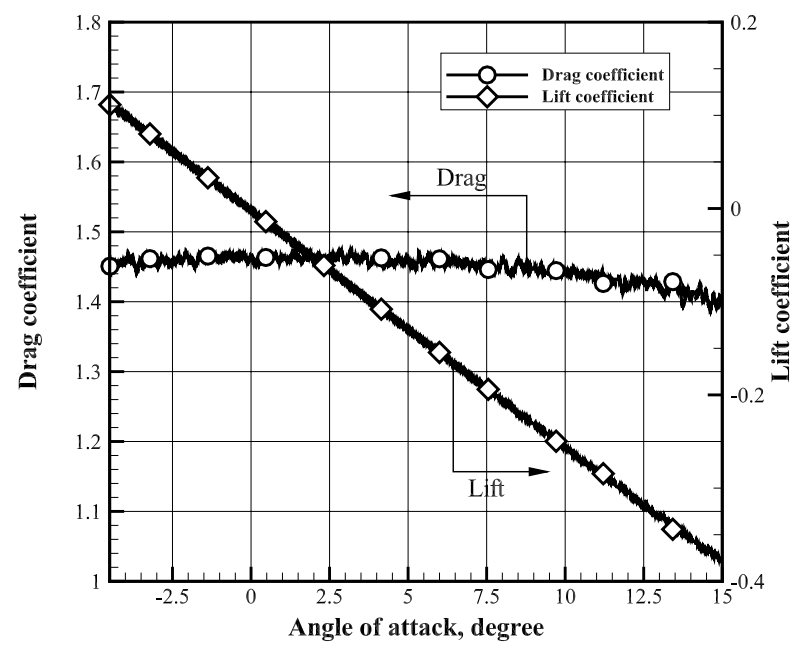

Figure 12: Drag and lift coefficients profiles relative to the angle of attack for the test model with $86 \mathrm{~mm}$ diameter at the Mach number of 1.2 in the wind tunnel experiment.

1.25 and 1.21, and then temporarily disappear below the Mach number of 1.20. However, a new reflected shock wave is formed on the sting backward at a Mach number of 1.17 once, and then disappears again at the Mach number of 1.05. The Mach number at which the reflected shock wave appears and disappears is different in each test model, although schlieren images for the other test model cases are not presented here. With respect to the reflected shock wave, the rear pressures change in a certain Mach number range and the drag coefficient then transiently changes. This is the main reason why the scale effect resulting in the local minimum value of the drag coefficient is observed in the supersonic region.

\subsection{Grid Study}

To investigate the independence of the computational grids used in the CFD approach, numerical simulations for the grid study are performed in the case of the test model with 86 mm diameter with sting and a Mach number of 1.2. The angle of attack is set to 5०. A fine computational grid system with 19,096,604 cells and 3,235,946 nodes is used. The normal grid system has 5,444,556 cells and 969,497 nodes, which is almost the same resolution as that of the grid adopted for the cases with an angle of attack of $0 \circ$. The comparisons of the computed drags and measured data are listed in Table 3. The error in the drag coefficients generated due to the use of normal and fine grids is approximately one percent. This result indicates that the normal computational grids are sufficiently convergent.

Table 3: Comparison of drag coefficients for the test model with $86 \mathrm{~mm}$ diameter given a Mach number of 1.2 with respect to the normal and fine grids and experimental data.

\begin{tabular}{ccc}
\hline \hline CFD (Normal) & CFD (Fine) & Exp. \\
\hline 1.492 & 1.477 & 1.457 \\
\hline \hline
\end{tabular}




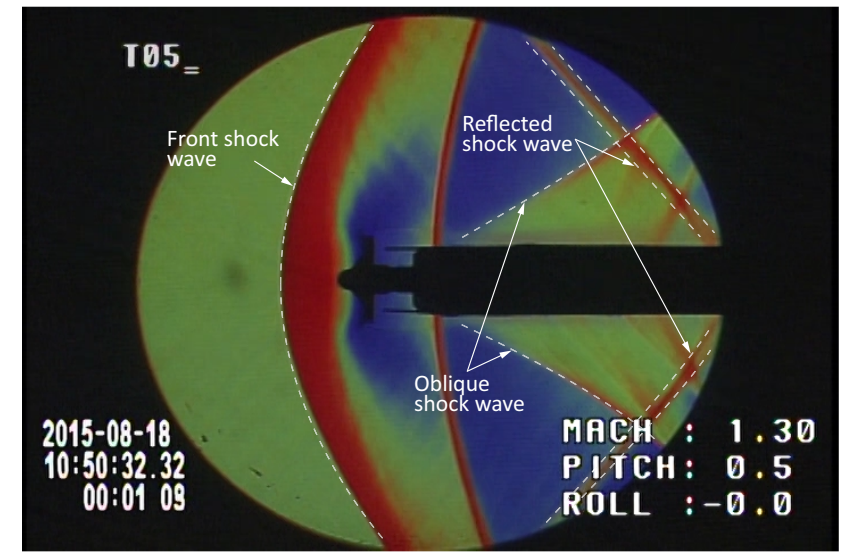

(a) Mach number 1.30

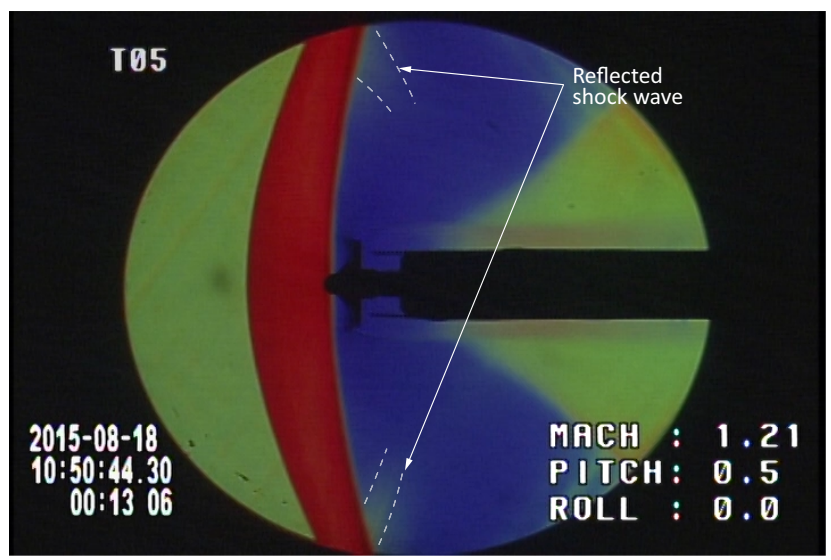

(c) Mach number 1.21 .

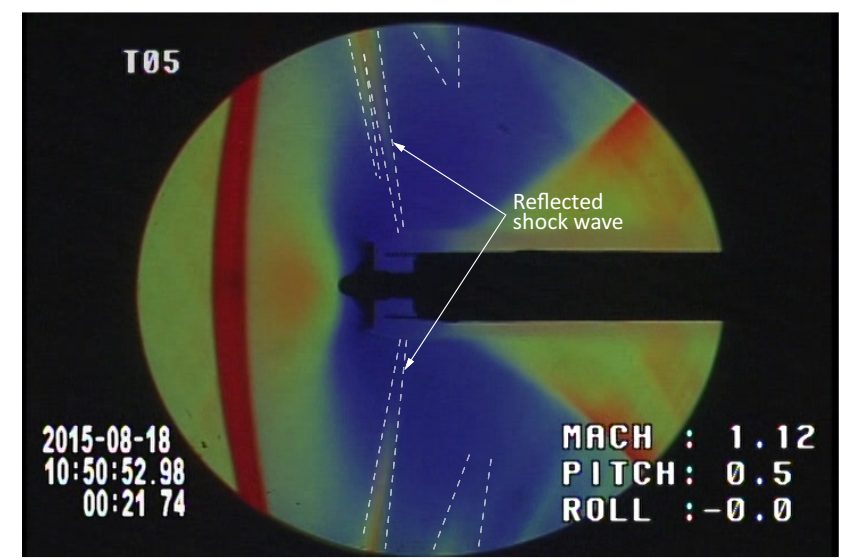

(e) Mach number 1.12 .

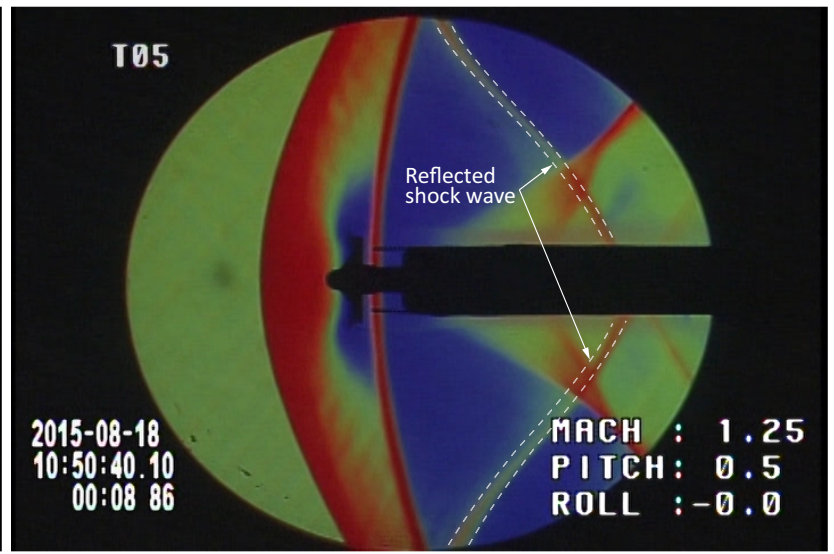

(b) Mach number 1.25.

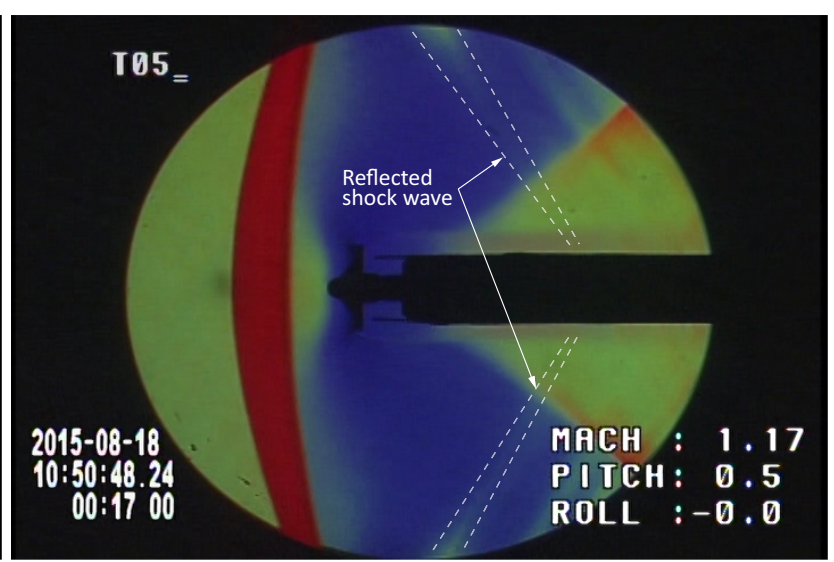

(d) Mach number 1.17 .

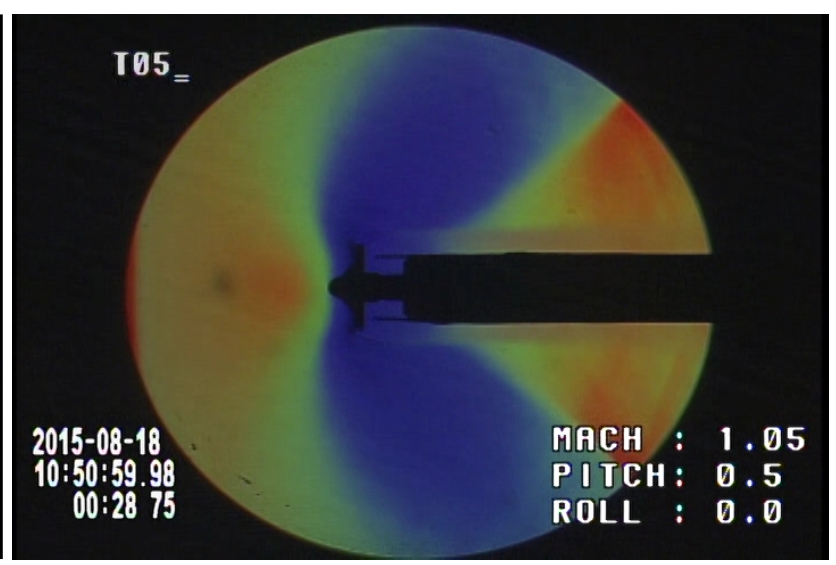

(f) Mach number 1.05 .

Figure 13: Schlieren images around the test model of $66 \mathrm{~mm}$ in diameter. 


\subsection{Sting Effect}

Computations of the transonic flow around each test model are performed for the freestream conditions listed in Table 1. Figure 14 shows a comparison of the drag coefficient profiles for the test models with diameters of 86 and $66 \mathrm{~mm}$ between the experiment and CFD results with/without sting. Additionally, Fig. 15 shows a comparison of the rear pressure coefficient profiles for the test models with diameters of 86 and $66 \mathrm{~mm}$ between the experimental and CFD results with/without sting. The CFD results are obtained under the condition of no angle of attack. The computed pressure corresponds to an arithmetically averaged value by using values obtained at the four positions arranged in the rear of the test model in the same manner as the experiment.

The CFD results with the sting exhibit good agreement with the experimental data. Prediction performance of the present analysis model is sufficiently reasonable. In contrast, the CFD results do not reproduce the local minimum values of the drag coefficient in the supersonic region. This suggests that the local minimum values are attributed to the effect of shock waves reflected on the wind tunnel wall, which is not considered in the present model analysis as discussed earlier.

With respect to the CFD cases without sting, steep decreases in the drag, such as the SMAAC flight data, are confirmed at a Mach number of 1, while gradual decreases, such as the experiment data, appear for the case with the sting. The difference in the computed drags between the sting and no-sting cases increases when the test model size decreases. This implies that the effect of the sting is relatively higher for the smaller test model, given that the sting diameter is the same for all test models. This indicates that the difference in the drag coefficient between the wind tunnel and the SMAAC flight test mainly occurs in the presence of the sting in the experiment. Moreover, the effect of the Reynolds number is one of the attributions to the discrepancy in the drag coefficients between the computed results and the SMAAC flight test results.

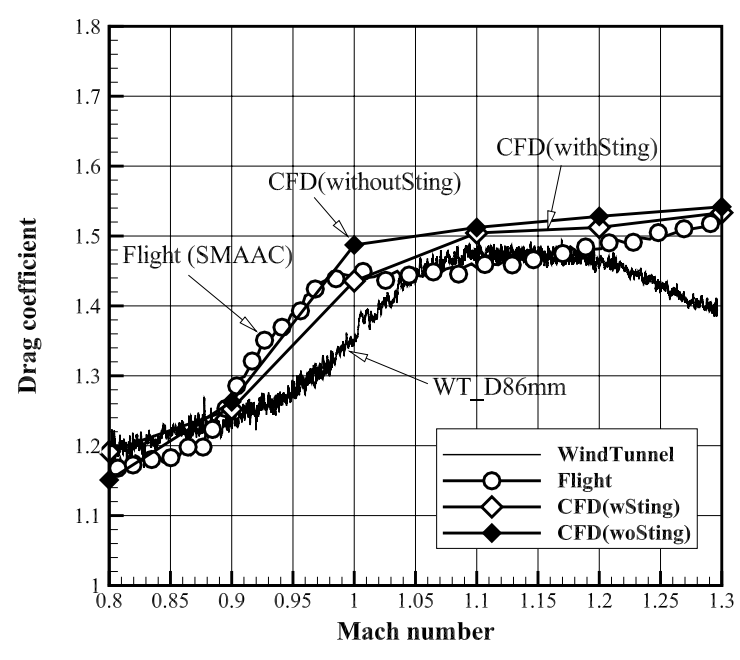

(a) Test model of $86 \mathrm{~mm}$ in diameter.

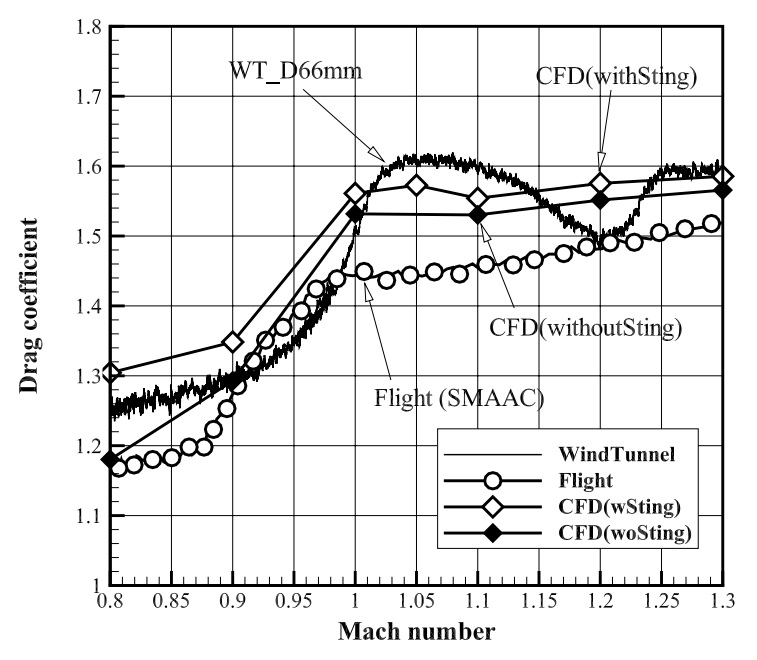

(b) Test model of $66 \mathrm{~mm}$ in diameter.

Figure 14: Comparison of drag coefficient profiles among experiment, flight test, and CFD for cases with/without sting. 


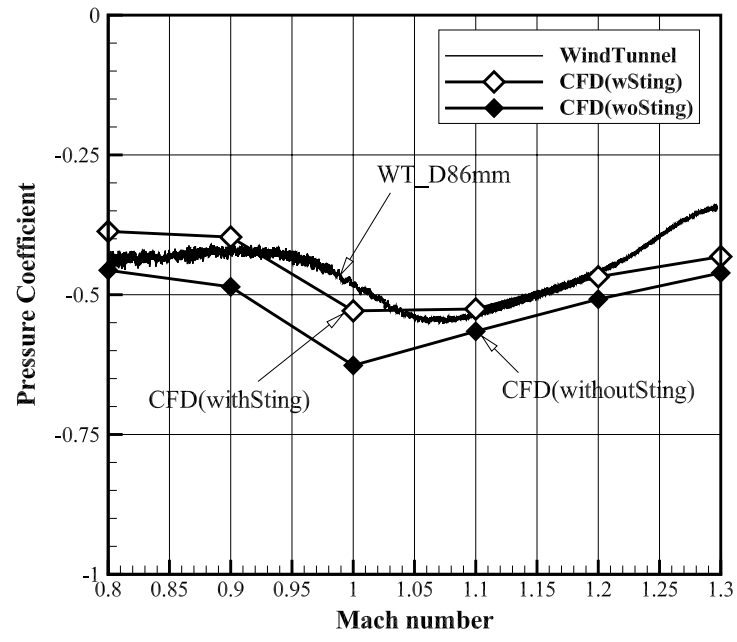

(a) Test model of $86 \mathrm{~mm}$ in diameter.

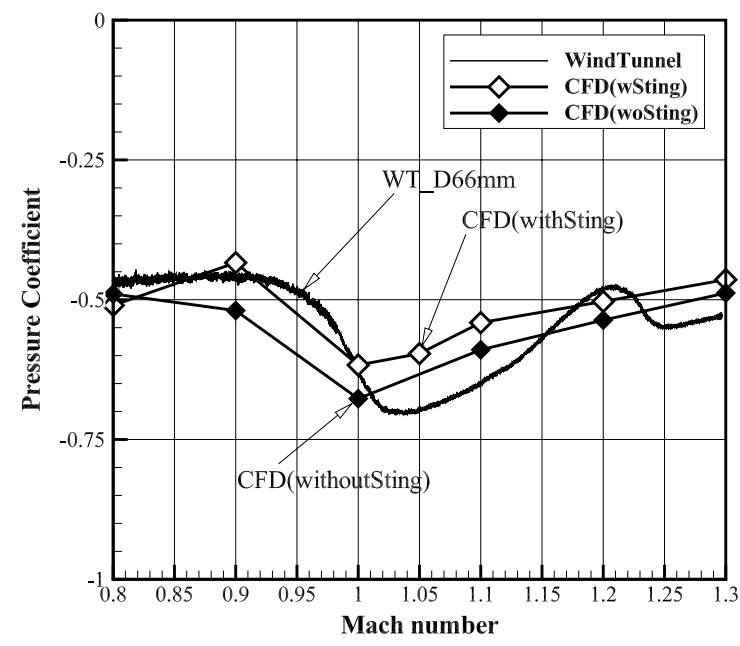

(b) Test model with $66 \mathrm{~mm}$ diameter.

Figure 15: Comparison of pressure coefficient profiles between the experiment and CFD for cases with/without sting.

\subsection{Wake Structure}

Curved vectors of velocity around the test model with $86 \mathrm{~mm}$ diameter for a Mach number of 0.9 with and without sting are shown in Figs. 16(a) and 16(b), respectively. Note that the RANS model is used in the analytical model and that the computed flow fields are results of time-averaged flow. The flow structure in the wake mainly changes due to the sting behind the test model. For the case with sting, a large vortex is formed near the sting by separation on the torus. A secondary vortex is induced by the vortex near the sting. Conversely, a large ring-shaped vortex is generated behind the test model for the case without a sting. The ring-shaped vortex is also confirmed in the CFD results [19] of SMAAC flight. Rear pressure without sting becomes lower than that with sting due to the strong vortex behind the vehicle (also shown in Fig. 15). The contribution of rear pressure to the drag is enhanced below the sonic velocity because the compressibility effect on the front-face pressure reduces. This indicates that the difference between these wake structures with/without sting arises due to the difference in drag behaviors for the transonic regime.

The predicted drag coefficients for the test model with $86 \mathrm{~mm}$ diameter without sting are lower than those of the 66-mm-diameter test model, as shown in Fig. 14, under all considered speeds. The discrepancy is mainly attributed to the effect of Reynolds number, i.e., scale effect. Turbulence in the wake is enhanced for a larger scale model case and leads to high pressure behind the test model. This is also confirmed in the CFD results for the SMAAC flight test [25].

\section{Conclusions}

In this study, the drag behavior of an inflatable reentry vehicle at transonic speeds was investigated using a transonic wind tunnel and the computational fluid dynamics (CFD) approach. Five test models involving diameters ranging between 96 and $56 \mathrm{~mm}$ were used with a test model to evaluate the scale effect. The shapes of the five test models were 


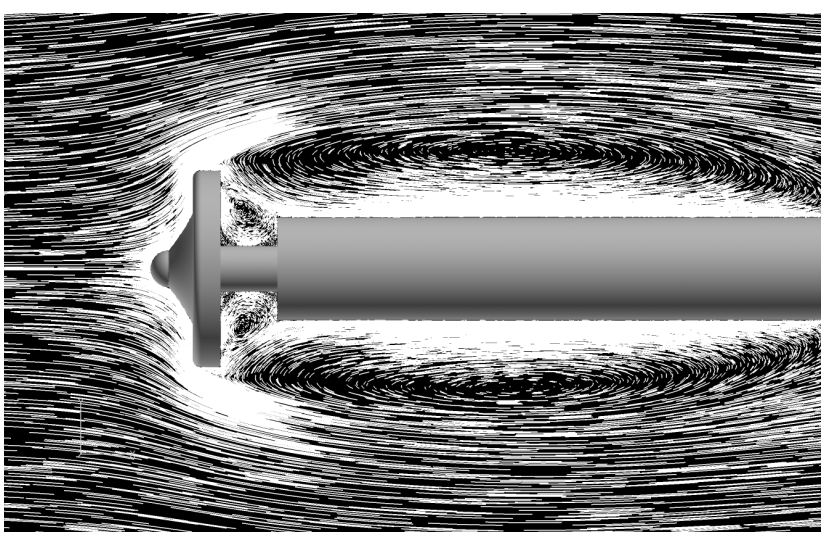

(a) Case with sting.

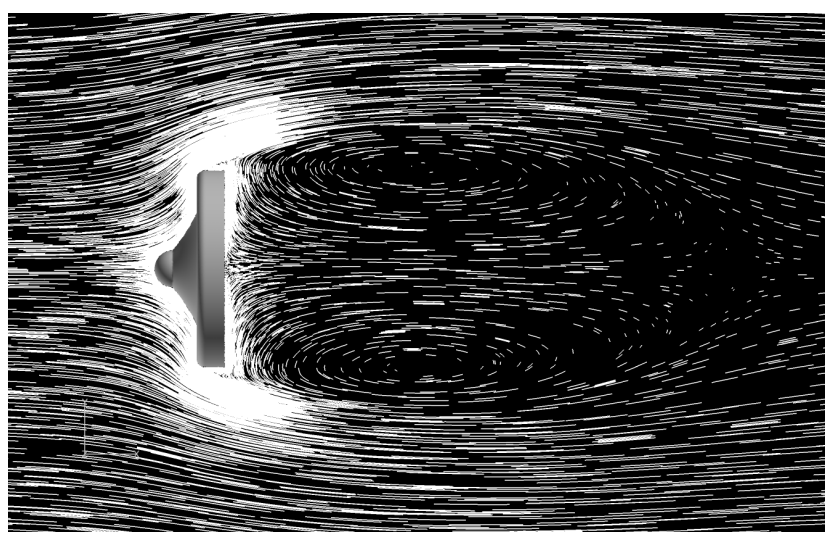

(b) Case without sting.

Figure 16: Velocity vectors for a test model with a diameter of $86 \mathrm{~mm}$ given a Mach number of 0.9 .

essentially similar with that of a reentry vehicle of suborbital reentry demonstration, except for the head capsule and torus tube. Additionally, a test model with different rear-side shapes was used to investigate the rear shape effect.

Aerodynamic forces, density gradient distributions, and pressures in the rear of the test models were measured in the wind tunnel experiment. The drag coefficient profiles relative to the Mach number were compared with the results of suborbital reentry demonstration. The results indicated the discrepancy of the drag coefficient between the wind tunnel and the flight test. Furthermore, steep decreases that were observed in the profile of the drag coefficient with decreases in the Mach number in the flight test became smooth in the wind tunnel experiments. Moreover, the wind tunnel experiments showed local minimum values of the drag coefficient in the supersonic regime. The results indicated that the rear shape does not significantly influence aerodynamics in a transonic wind tunnel.

The CFD technique was used, and reasons behind the behaviors of the drag coefficient were investigated in detail. The results indicated that the sting effect behind the test models results in smoothed profiles in the transonic regime, and that a decrease in the scale of the test model leads to a relative increase in the sting effect. Furthermore, the shock waves reflected on the test-section wall of the wind tunnel are attributed to the local minimum values in the supersonic regime.

\section{Acknowledgments}

This study was supported by JSPS KAKENHI Grant Numbers 15H04205 and 17K14871. The present computational results were obtained by using the fast unstructured CFD code FaSTAR, which was developed by JAXA. This study was partially supported by "Joint Usage/Research Center for Interdisciplinary Large-scale Information Infrastructures" and "High Performance Computing Infrastructure" in Japan (Project ID: jh160032 and jh170047). The computations were performed by using the computational facilities (HITACHI HA8000tc/HT210 and FUJITSU PRIMERGY CX400) at the Research Institute for Information Technology, Kyushu University, and the supercomputer system (HITACHI SR16000 model M1) at the Information Initiative Center, Hokkaido University. 


\section{References}

[1] M. Gräßilin and U. Schöttle. "Flight Performance Evaluation of the Reentry Mission IRDT-1". In Papers Presented at the 52nd International Astronautical Congress, IAF Paper 01-V305, Toulouse, France, October 1 - 52001.

[2] S.J. Hughes, R.A. Dillman, B.R. Starr, R.A. Stephan, M.C. Lindell, C.J. Player, and D.F.M. Cheatwood. "Inflatable Re-entry Vehicle Experiment (IRVE) Design Overview". AIAA Paper 2005-1636, 2005.

[3] R.R. Rohrschneider and R.D. Braun. "A Survey of Ballute Technology for Aerocapture". Journal of Spacecraft and Rockets, 44(1):10-23, January - February 2007.

[4] P. Reynier and D. Evans. "Postflight Analysis of Inflatable Reentry and Descent Technology Blackout During Earth Reentry". Journal of Spacecraft and Rockets, 46(4):800-809, July-August 2009.

[5] S.J. Hughes, D.F.M. Cheatwood, A.M. Calomino, and H.S. Wright. "Hypersonic Inflatable Aerodynamic Decelerator (HIAD) Technology Development Overview". AIAA Paper 2011-2524, 2011.

[6] T. Abe. "A Self-Consistent Tension Shell Structure for Application to Aerobraking Vehicle and Its Aerodynamic Characteristics". AIAA Paper 1988-3405, 1988.

[7] I.G. Clark, A.L. Hutchings, C.L. Tanner, and R.D. Braun. "Supersonic Inflatable Aerodynamic Decelerators for Use on Future Robotic Missions to Mars". Journal of Spacecraft and Rockets, 46(2):340-352, March - April 2009.

[8] K. Yamada, D. Akita, E. Sato, K. Suzuki, T. Narumi, and T. Abe. "Flare-Type Membrane Aeroshell Flight Test at Free Drop from a Balloon". Journal of Spacecraft and Rockets, 46(3):606-614, May-June 2009.

[9] K. Yamada, T. Abe, K. Suzuki, N. Honma, M. Koyama, Y. Nagata, D. Abe, Y. Kimura, A.K. Hayashi, D. Akita, and H. Makino. "Deployment and Flight Test of Inflatable Membrane Aeroshell using Large Scientific Balloon". AIAA Paper 2011-2579, 2011.

[10] K. Yamada, M. Koyama, Y. Kimura, K. Suzuki, T. Abe, and A.K. Hayashi. "Hypersonic Wind Tunnel Test of a Flare-type Membrane Aeroshell for Atmospheric Entry Capsule". ISTS Special Issue: Selected papers from the 27th ISTS, Transactions of JSASS, 7(ists27):27-32, 2010.

[11] K. Yamada, T. Sonoda, K. Nakashino, and T. Abe. "Structural Strength of Flare-type Membrane Aeroshell Supported by Inflatable Tours against Aerodynamic Force". In Proceedings of 28th International Symposium on Space Technology and Science, ISTS 2011-c-34, Okinawa, Japan, June 5 - 122011.

[12] Y. Takahashi, K. Yamada, and T. Abe. "Examination of Radio Frequency Blackout for an Inflatable Vehicle during Atmospheric Reentry". Journal of Spacecraft and Rockets, 51(2):430-441, March 2014.

[13] K. Yamada, T. Abe, K. Suzuki, O. Imamura, and D. Akita. "Reentry Demonstration Plan of Flare-type Membrane Aeroshell for Atmospheric Entry Vehicle using a Sounding Rocket". AIAA Paper 2011-2521, 2011. 
[14] K. Yamada, Y. Nagata, T. Abe, K. Suzuki, O. Imamura, and D. Akita. "Suborbital Reentry Demonstration of Inflatable Flare-Type Thin-Membrane Aeroshell Using a Sounding Rocket". Journal of Spacecraft and Rockets, 52(1):275-284, February-March 2015 .

[15] K. Yamada, Y. Nagata, T. Abe, K. Suzuki, O. Imamura, and D. Akita. "Reentry Demonstration of Flare-type Membrane Aeroshell for Atmospheric Entry Vehicle using a Sounding Rocket". AIAA Paper 2013-1388, 2013.

[16] K. Yamada, Y. Nagata, N. Honma, D. Akita, O. Imamura, T. Abe, and K. Suzuki. "Reentry Demonstration Deployable and Flexible Aeroshell for Atmospheric-Entry Vehicle using Sounding Rocket". In Proceedings of 63th International Astronautical Congress, AC-12-D2.3.3, Naples, Italy, October 1 - 52012.

[17] Y. Nagata, K. Yamada, T. Abe, and k Suzuki. "Attitude Dynamics for Flare-type Membrane Aeroshell Capsule in Reentry Flight Experiment". AIAA Paper 2013-1285, 2013.

[18] Y. Takahashi, K. Yamada, T. Abe, and K. Suzuki. "Aerodynamic Heating around Flaretype Membrane Inflatable Vehicle in Suborbital Reentry Demonstration Flight". Journal of Spacecraft and Rockets, 52(6):1530-1541, Novermber 2015.

[19] D. HA, Y. TAKAHASHI, K. YAMADA, and T. ABE. "Aerodynamic Simulation of Inflatable Re-Entry Vehicle Performance in Low Speed Wind Tunnel". TRANSACTIONS OF THE JAPAN SOCIETY FOR AERONAUTICAL AND SPACE SCIENCES, AEROSPACE TECHNOLOGY JAPAN, 12(ists29):Po_2_57-Po_2_62, 2014.

[20] K. Yamada, Y. Kato, and T. Abe. "Numerical Simulation of Hypersonic Flow around Flare-Type Aeroshell with Torus Frame". In Proceedings of $6^{\text {th }}$ Asia Workshop on Computational Fluid Dynamics, Kashiwa, Japan, March 162009.

[21] F.R. Menter, M. Kuntz, and R. Langtry. "Ten Years of Industrial Experience with the SST Turbulence Mode". Turbulence, Heat and Mass Transfer 4, ed: K. Hanjalic, Y. Nagano, and M. Tummers, Begell House, Inc., pages 625-632, 2003.

[22] Y. Takahashi. "Advanced validation of CFD-FDTD combined method using highly applicable solver for reentry blackout prediction". Journal of Physics D: Applied Physics, 49(1):015201, 2016.

[23] A. Hashimoto, K. Murakami, T. Aoyama, K. Ishiko, M. Hishida, M. Sakashita, and P.R. Lahur. "Toward the Fastest Unstructured CFD". AIAA Paper 2012-1075, 2012.

[24] E. Shima and K. Kitamura. "Parameter-Free Simple Low-Dissipation AUSM-Family Scheme for All Speeds". AIAA Journal, 49(8):1693-1709, August 2011.

[25] Y. Takahashi, D. Ha, N. Oshima, K. Yamada, T. Abe, and K. Suzuki. "Aerodecelerator Performance of Flare-Type Membrane Inflatable Vehicle in Suborbital Reentry". Journal of Spacecraft and Rockets, 54(5):993-1004, 2017. 